\title{
The Role of Error in the Conceptual Design of a Transport Aircraft
}

\author{
Robert A. McDonald*
}

\begin{abstract}
A fidelity trade environment was demonstrated by using it to simulate a decision making process for a transport aircraft. This scenario was not possible without the fidelity trade environment. The role of system feedback and coupling in error stability was also investigated.

A sensitivity approach which relies on the system sensitivity matrix was used to rapidly approximate the propagation of error through the complex system. In verification tests, the sensitivity approach provided approximate results substantially similar to a Monte Carlo approach that was many orders of magnitude more expensive. The rapid sensitivity approach to modeling error propagation enabled the responsive analysis required for an interactive environment.

A notional transport aircraft was modeled in the fidelity trade environment. The system was decomposed and the fidelity trade environment was used to integrate the system. Then, a scenario was described where a decision maker used the fidelity trade environment at the beginning of a complex systems design problem. Using the environment, the designer was able to make design decisions while considering error and he was able to make decisions regarding required tool fidelity as the design problem continues. These decisions could not be made in a quantitative manner before the fidelity trade environment was developed.

The role of system feedback and coupling in the growth or decay of an individual error source was investigated. The diagonal entry of the inverse system sensitivity matrix was identified as the determining factor in the stability of a particular error source.
\end{abstract}

\section{Introduction, Motivation, \& Background}

Complex man-made systems are ubiquitous in modern technological society. The national air transportation infrastructure and the aircraft that operate within it, the highways stretching coast-to-coast and the vehicles that travel on them, and global communications networks and the computers that make them possible are all complex systems.

It is impossible to fully validate a systems analysis or a design process. Systems are too large, complex, and expensive to build test and validation articles. Furthermore, the operating conditions throughout the life cycle of a system are impossible to predict and control for a validation experiment. Often, designers are interested in revolutionary systems for which there is no historical counterpart which can be used for validation.

Error is introduced at every point in a complex systems design process. Every error source propagates through the complex system in the same way information propagates. If a system has feedforward, the errors feed forward. If a system has feedback, then errors feed back. If a system has coupled loops, then errors are coupled.

As with error propagation through a single analysis, error sources grow and decay when propagated through a complex system. These behaviors are made more complex by the complex interactions of a complete system. This complication and the loss of intuition that accompanies it makes proper error propagation calculations even more important to aid the decision maker.

Error allocation and fidelity trade decisions answer questions like: Is the fidelity of a complex systems analysis adequate, or is an improvement needed? If an improvement is needed, how is that improvement best achieved? Where should limited resources be invested for the improvement of fidelity? How does knowledge

*Assistant Professor, Aerospace Engineering, Cal Poly San Luis Obispo, AIAA Member 
of the imperfection of a model impact design decisions based on the model? How does this knowledge impact the choice and certainty of the design point? How does it impact the certainty of the performance of a particular design?

A fidelity trade environment has been developed ${ }^{1,2}$ to provide the designer with a tool for understanding the flow and interaction of error through a complex system. The designer can use this environment to make quantitatively informed decisions regarding error to best improve the system model and thereby the design. In this paper, the environment was applied to the design of a typical wide-bodied transport aircraft. The aircraft model used in this paper is extremely simple, which allows the model to clearly demonstrate the impact of error at this stage of the design process.

The creation of a fidelity trade environment relied on the support of enabling techniques from complex systems design. Among these enabling techniques are error propagation, metamodeling, and information management.

\section{Error Propagation}

In addition to error inherent to a calculation, in any calculation the error in any input quantity contributes to an erroneous output. The magnitude of error in an output may be diminished or amplified relative to the error of the inputs. In a series of calculations representing a complex system, error can build up and interact in non-intuitive ways. The behavior of error becomes even more obscure when the series of calculations are coupled.

Error propagation is a set of techniques used to quantify and understand the error in an output quantity based on some knowledge of the error of the input quantities and error introduced during the process. Error propagation was originally developed for the experimental sciences. ${ }^{3,4}$ In an experiment, the source of all error is measurement, as Mother Nature makes no mistakes. Conversely, computer analysis codes have many sources of error. They are subject to error in their inputs and their process but there is no measurement error in their outputs. ${ }^{5,6}$

A sensitivity approach was used to rapidly approximate the propagation of error through complex systems. Please refer to the author's preceding paper ${ }^{2}$ or Ph.D. thesis ${ }^{1}$ for a more complete discussion of the error propagation formulation. This approach relies on the system sensitivity matrix ${ }^{7}$ to model the behavior of a complex system as a whole. The system sensitivity matrix proposed by Sobieski contains the partial derivatives which govern the interaction of tasks within a complex system. The form of the system sensitivity matrix (M) used in this work is given below.

$$
\mathbf{M}=\left[\begin{array}{cccc}
\mathbf{I}_{1} & -\frac{\partial \mathbf{g}_{1}}{\partial \mathbf{g}_{2}} & \cdots & -\frac{\partial \mathbf{g}_{1}}{\partial \mathbf{g}_{l}} \\
-\frac{\partial \mathbf{g}_{2}}{\partial \mathbf{g}_{1}} & \mathbf{I}_{2} & & \vdots \\
\vdots & & \ddots & -\frac{\partial \mathbf{g}_{l-1}}{\partial \mathbf{g}_{l}} \\
-\frac{\partial \mathbf{g}_{l}}{\partial \mathbf{g}_{1}} & \cdots & -\frac{\partial \mathbf{g}_{l}}{\partial \mathbf{g}_{l-1}} & \mathbf{I}_{l}
\end{array}\right]
$$

The propagation of random error sources through a complex system is governed by Equation 2 given below. This equation first combines the input error sources $\left(\sigma_{\mathbf{x}}\right)$ and the task local error sources $\left(\varsigma_{\mathbf{f}}\right)$. It then uses the inverse system sensitivity matrix $\left(\mathbf{M}^{-1}\right)$ to map these errors into the propagated global error $\left(\sigma_{\mathbf{f}}\right)$. This equation is approximate only in that it represents a linearization for the case of finite error sources. Note that the square operations in Equation 2 are performed on a term by term basis. Although the notation and approach to error propagation is different from that of $\mathrm{Du}$ and $\mathrm{Chen},{ }^{8}$ the resulting equations are equivalent.

$$
\sigma_{\mathbf{f}}^{2} \approx\left(\mathbf{M}^{-1}\right)^{2}\left[\varsigma_{\mathbf{f}}^{2}+\left(\frac{\partial \mathbf{f}}{\partial \mathbf{x}}\right)^{2} \sigma_{\mathbf{x}}^{2}\right]
$$

In verification tests, the sensitivity approach has provided approximate results substantially similar to a Monte Carlo approach that was many orders of magnitude more expensive. The rapid sensitivity approach to modeling error propagation enabled the responsive analysis required for an interactive environment. One such verification test was included later in this paper. 


\section{Transport Aircraft Model}

This complex system model is made of a series of tasks, each pertaining to a classical discipline of aerospace engineering. Each task defines an interface, a set of inputs and outputs. Any analysis which implements the task interface may be used in the system study. Simple implementations of the task interfaces were developed as prototype analyses; these algebraic approximations could be considered "textbook" methods. The fidelity trade analysis can then be used to identify which task implementations must be improved. Although the discussion here is necessarily brief, this example has been fully documented by the author. ${ }^{1}$ Any quantities not specified as outputs from a task are treated as system level inputs. The resulting system is shown in Figure 1.

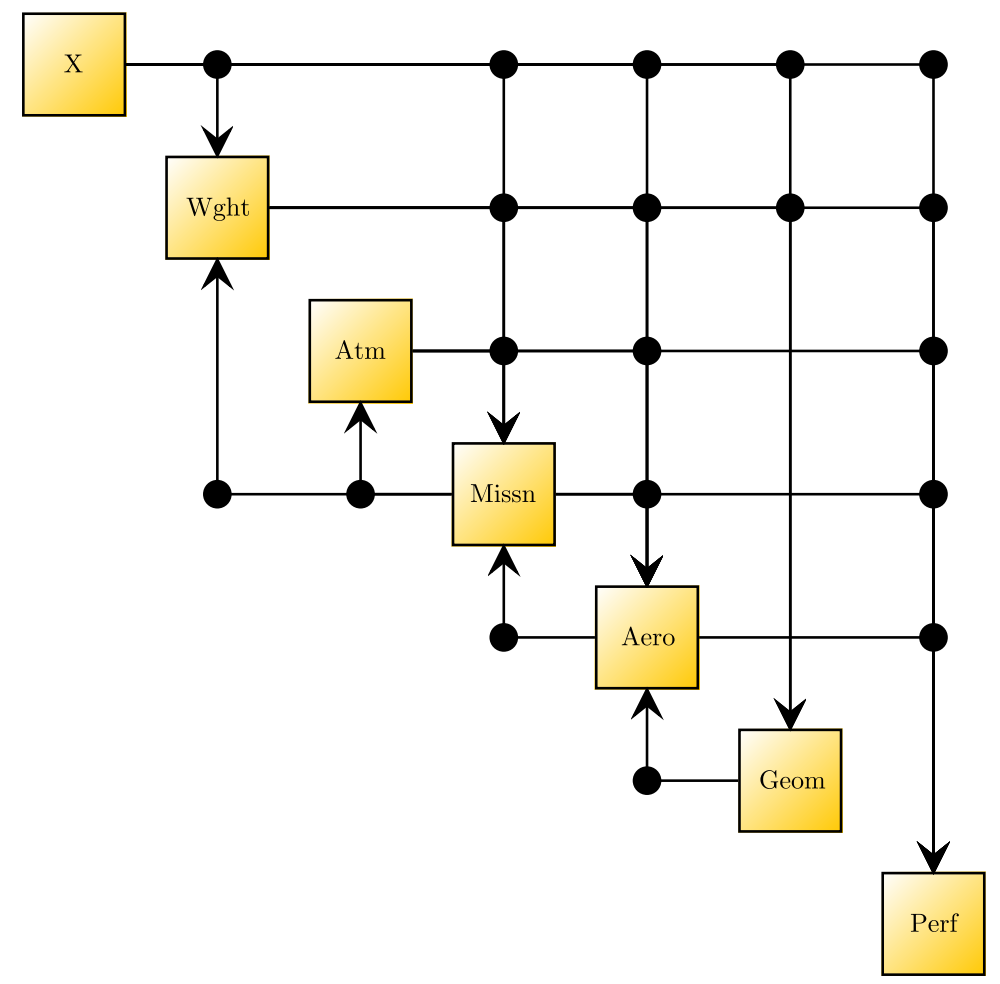

Figure 1. Example system DSM

Ranges of interest and baseline values for the system level inputs were selected to represent a large design space of alternatives as listed in Table 1(a).

Additionally, some quantities that would qualify as system level variables were set to representative values and held constant throughout the study. This effectively hides these quantities from the systems perspective. This was done to limit the scope of the study and the resulting visualizations to a manageable level. These implied variables and their settings are listed in Table 1(b).

The baseline settings of the system level variables result in a converged vehicle represented by the system outputs listed in Table 2(a). A three-dimensional model of the baseline aircraft is given in Figure 2.

A system explorer view of the entire aircraft design space is included as Figure 3. The hairlines represent the baseline values of the system level variables. The curves represent the variation of the system response to the change of a single system level variable. Each point contributing to a curve in the system explorer represents a converged vehicle model.

Some representative constraints were placed on the point performance of the aircraft as listed in Table 2(b). These constraints were plotted on a customary $T / W$ vs. $W / S$ view of the design space as shown in Figure 4(a). The vertical and horizontal hairlines represent the baseline vehicle design point. As expected, the baseline vehicle satisfies the constraints. 
Table 1. System level variable ranges and settings

(a) Explicit variable settings

\begin{tabular}{ccccc}
\hline \hline Quantity & Min. & Max. & Baseline & Units \\
\hline$W_{p}$ & 10,000 & 100,000 & 55,000 & $l b f$ \\
$W / S$ & 50 & 150 & 100 & $l b f / f t^{2}$ \\
$l_{\text {fuse }}$ & 150 & 200 & 175 & $f t$ \\
$R$ & 6 & 12 & 9 & - \\
$S F C$ & 0.3 & 0.7 & 0.5 & $l b m / h r / l b f$ \\
$R$ & 3000 & 9000 & 6000 & $n m i$ \\
$W_{f, a d d} / W_{f}$ & 5 & 30 & 17.5 & $\%$ \\
$T / W$ & 0.18 & 0.3 & 0.24 & - \\
$C_{L, \max }$ & 1.0 & 4.0 & 2.5 & - \\
\hline \hline
\end{tabular}

(b) Implied variable settings

\begin{tabular}{lrl}
\hline \hline Quantity & Value & Units \\
\hline$M_{c r}$ & 0.83 & - \\
$\Lambda_{c / 2}$ & $25^{\circ}$ & \\
$D_{\text {fuse }}$ & 17 & $f t$ \\
$S_{V T} / S$ & 10 & $\%$ \\
$S_{H T} / S$ & 23 & $\%$ \\
$\lambda$ & 0.25 & - \\
$\tau$ & 12 & $\%$ \\
\hline \hline
\end{tabular}

Table 2. Aircraft characteristics

(a) Sized vehicle characteristics

\begin{tabular}{|c|c|c|c|c|c|}
\hline Quantity & Value & Units & Quantity & Value & Units \\
\hline$W$ & 310000 & $l b f$ & $C_{D, 0 \mathrm{cr}}$ & 0.0151 & - \\
\hline$\sigma$ & 0.193 & - & $C_{D, 0 \mathrm{sl}}$ & 0.0113 & - \\
\hline$W_{f} / W$ & 34.5 & $\%$ & $e_{c r}$ & 0.735 & - \\
\hline$\theta$ & 0.752 & - & $e_{s l}$ & 0.655 & - \\
\hline$S_{w e t, w i n g}$ & 5325 & $f t^{2}$ & $S_{T O}$ & 5430 & $f t$ \\
\hline$S_{w e t, f u s e}$ & 7800 & $f t^{2}$ & $S_{L D G}$ & 4200 & ft \\
\hline$S_{w e t, a d d}$ & 2880 & $f t^{2}$ & $P_{s, s l}$ & 3360 & $\mathrm{ft} / \mathrm{min}$ \\
\hline & & & $P_{s, c r}$ & 390 & $\mathrm{ft} / \mathrm{min}$ \\
\hline
\end{tabular}

(b) Aircraft constraints

\begin{tabular}{llcl}
\hline \hline Quantity & & Value & Units \\
\hline$S_{T O}$ & $<$ & 6000 & $\mathrm{ft}$ \\
$S_{L D G}$ & $<$ & 4500 & $\mathrm{ft}$ \\
$P_{s, s l}$ & $>$ & 3000 & $\mathrm{ft} / \mathrm{min}$ \\
$P_{s, c r}$ & $>$ & 300 & $\mathrm{ft} / \mathrm{min}$ \\
\hline \hline
\end{tabular}




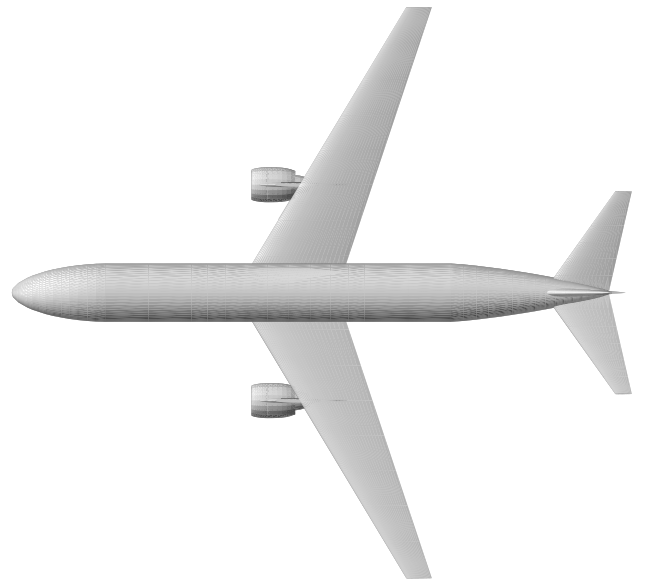

(a) Top

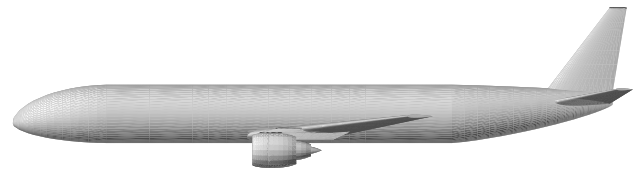

(c) Side

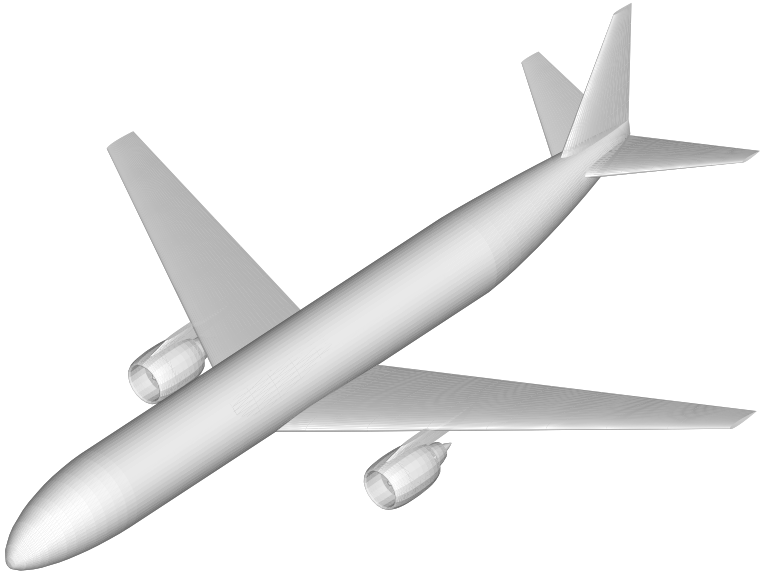

(b) Isometric

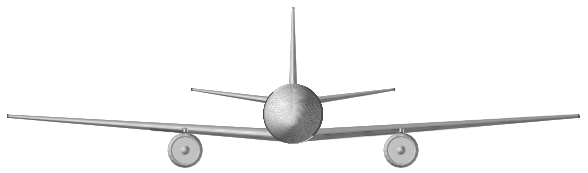

(d) Front

Figure 2. Baseline aircraft

\section{Error Management}

In order to demonstrate the utility of the fidelity trade environment, a representative error management scenario is carried out on the example transport aircraft system. The focus of this example is to illustrate a possible fidelity decision making process, so the point of interest and constraint settings representing the aircraft design, and therefore the design and size of the example vehicle, will not be changed. Of course, the environment is also capable of supporting more traditional decisions in design. This scenario serves as an experiment to test the effectiveness of the fidelity trade environment, in support of testing the research hypotheses.

In this scenario, a designer is at the start of a complex systems design process. The designer has built a system model based upon legacy codes. The designer is interested in accomplishing three major goals. First, the designer wants to choose a baseline design for the vehicle with an understanding of the impact of error on that choice. Second, the designer wants to calculate a preliminary estimate of the size and performance of the baseline design with some understanding of the accuracy of those estimates. Third, the designer wants to make decisions about the required analysis fidelity going forward in the design process. These fidelity decisions will guide investment and development in analysis tools. The fidelity trade environment should enable the designer to accomplish all of these goals.

The first step in the error management exercise is to introduce some representative sources of error. For problem simplicity and in order to highlight the differing impact of different error sources, the representative error sources are all introduced with the same magnitude, 5\%. Similarly, when a fidelity improvement is made, the error source is reduced to $1 \%$. While these error levels are not representative of the actual error of the methods used in the example problem, they illustrate the utility of fidelity trades without introducing the complexity of making and justifying error estimates for every component. The initial error levels applied are listed in Table $3(\mathrm{a})$.

The introductory propagated error breakdown for the system variables is included as Figure 5. Specific excess power at cruise shows a very large percent error; this is largely due to the relatively small magnitude of the excess power relative to the potential changes due to the error sources.

As a verification experiment, a Monte Carlo approach was used to propagate the error through the 


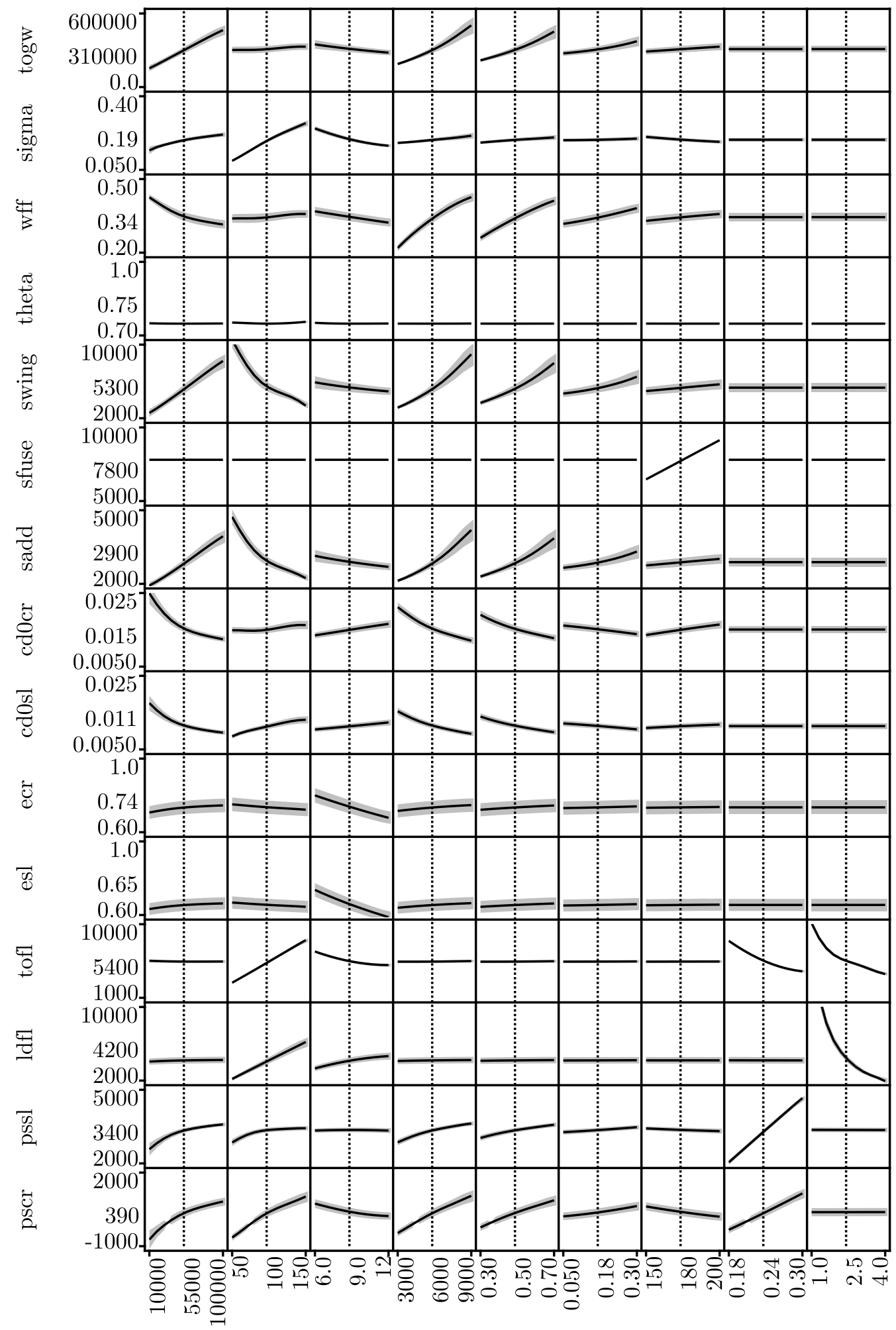

wp wos ar range sfccr wffadd lfuse tow clmax

Figure 3. Aircraft design space with introductory error 


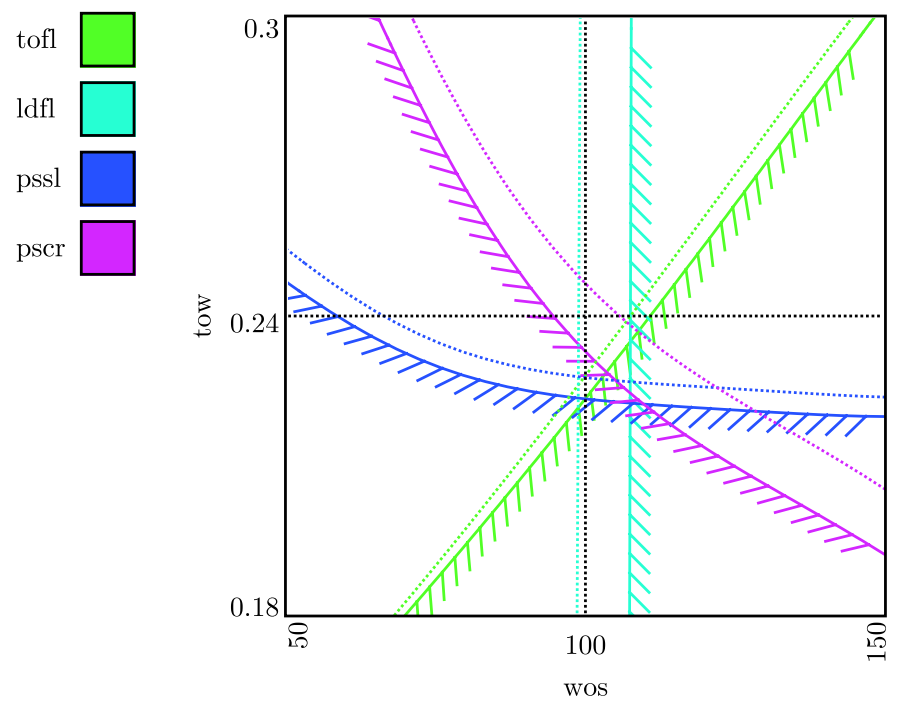

(a) Initial error

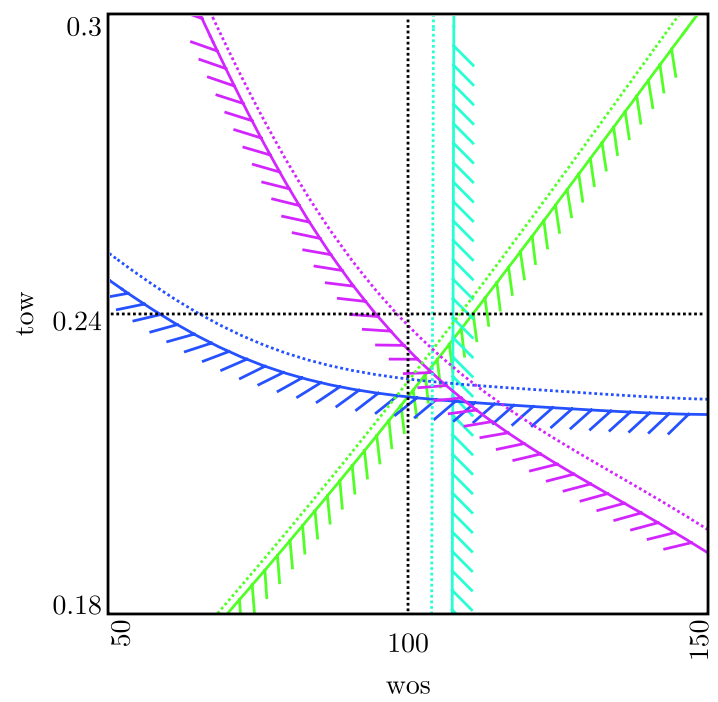

(c) Reduced error for cruise

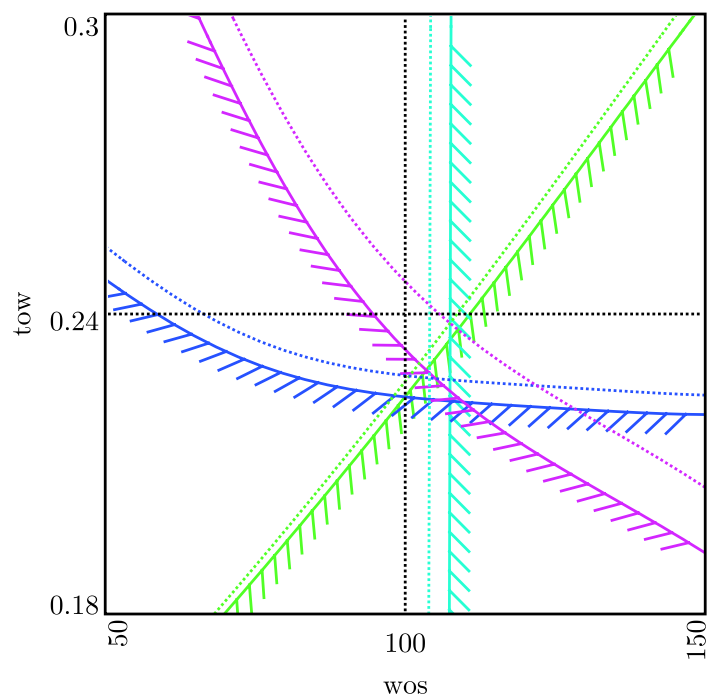

(b) Reduced error for landing

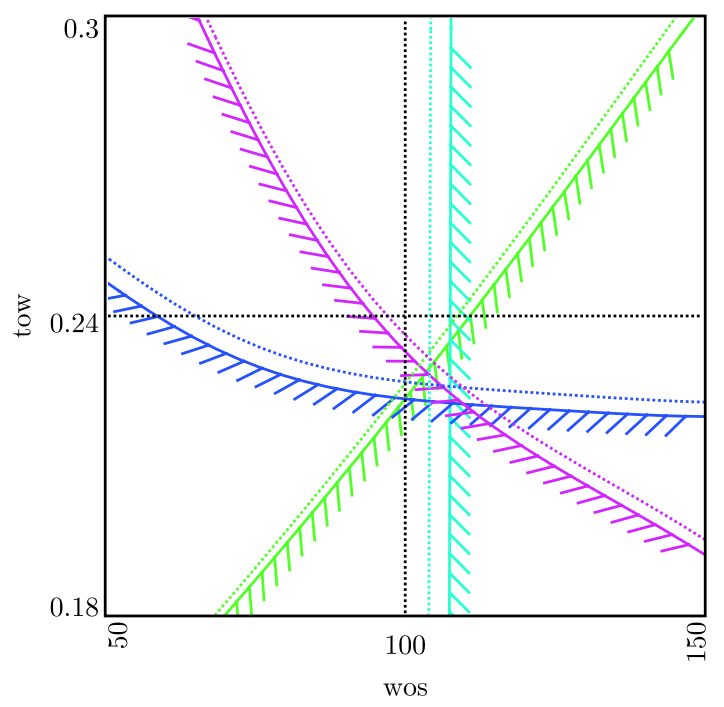

(d) Reduced error for weight

Figure 4. Aircraft constraints with propagated error 
Table 3. Error sources

\begin{tabular}{lcccc}
\hline \hline & $\begin{array}{c}\text { Initial Error } \\
\text { Quantity }\end{array}$ & \multicolumn{3}{c}{ Reduced Error for } \\
& & $\begin{array}{c}\text { (b) } \\
\text { Landing }\end{array}$ & $\begin{array}{c}\text { (c) } \\
\text { Cruise }\end{array}$ & $\begin{array}{c}\text { (d) } \\
\text { Weight }\end{array}$ \\
\hline$\sigma C_{L, \max }$ & $5 \%$ & $1 \%$ & $1 \%$ & $1 \%$ \\
$\varsigma e_{c r}$ & $5 \%$ & $5 \%$ & $5 \%$ & $5 \%$ \\
$\varsigma W$ & $5 \%$ & $5 \%$ & $5 \%$ & $1 \%$ \\
$\varsigma C_{D, 0} c r$ & $5 \%$ & $5 \%$ & $1 \%$ & $1 \%$ \\
$\sigma S F C$ & $5 \%$ & $5 \%$ & $1 \%$ & $1 \%$ \\
$\varsigma C_{D, 0} s l$ & $5 \%$ & $5 \%$ & $5 \%$ & $5 \%$ \\
$\varsigma e_{s l}$ & $5 \%$ & $5 \%$ & $5 \%$ & $5 \%$ \\
$\varsigma W_{f} / W$ & $5 \%$ & $5 \%$ & $1 \%$ & $1 \%$ \\
\hline \hline
\end{tabular}

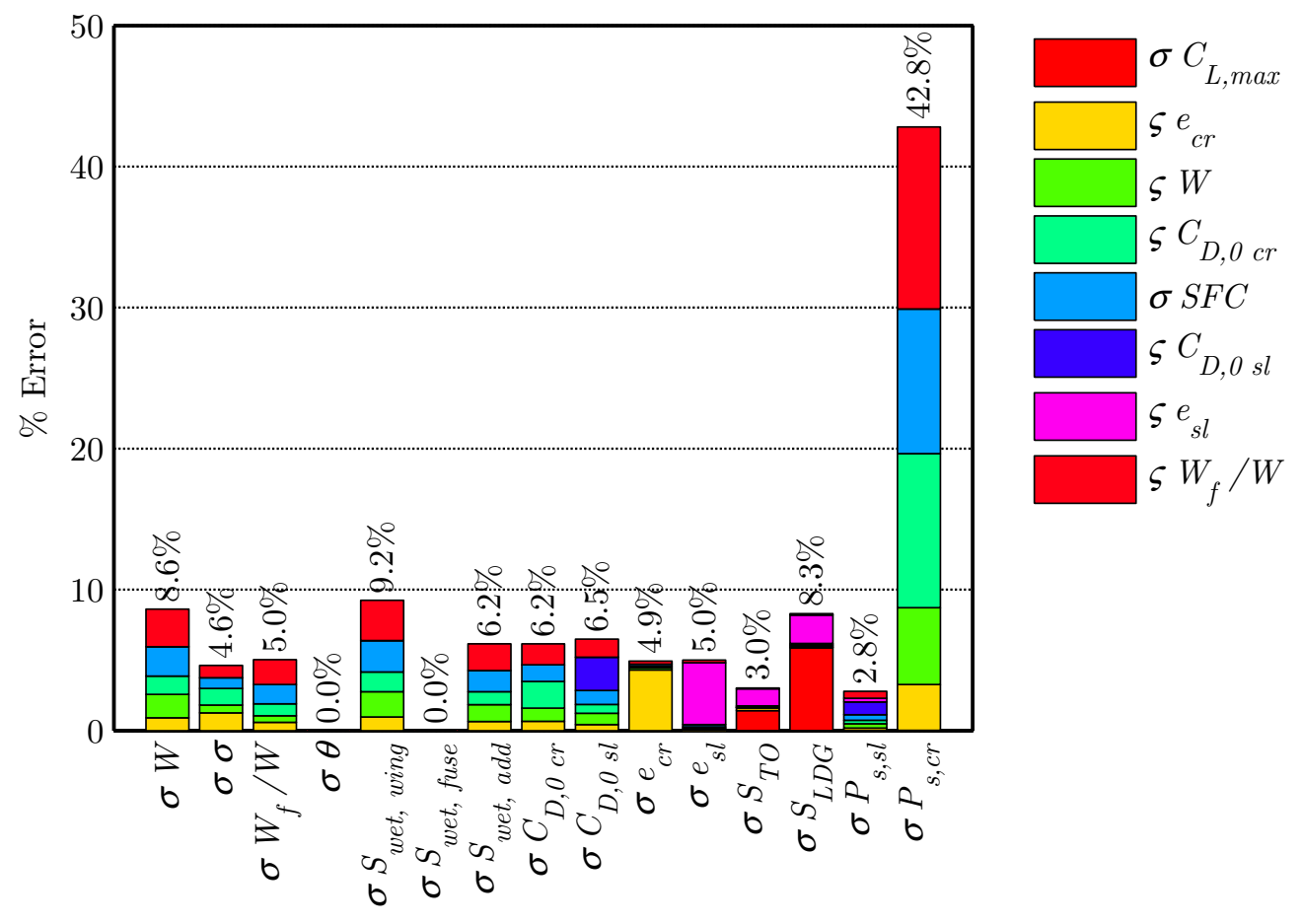

Response

Figure 5. Introductory error breakdown 
complex system. The results of the Monte Carlo analysis were compared with the sensitivity based approach in Figure 6. The blue and red bars represent the results of the sensitivity approach and the Monte Carlo approach respectively. The small error bands at the top of the Monte Carlo bar represent the one sigma error in the result due to the use of a finite sample size.

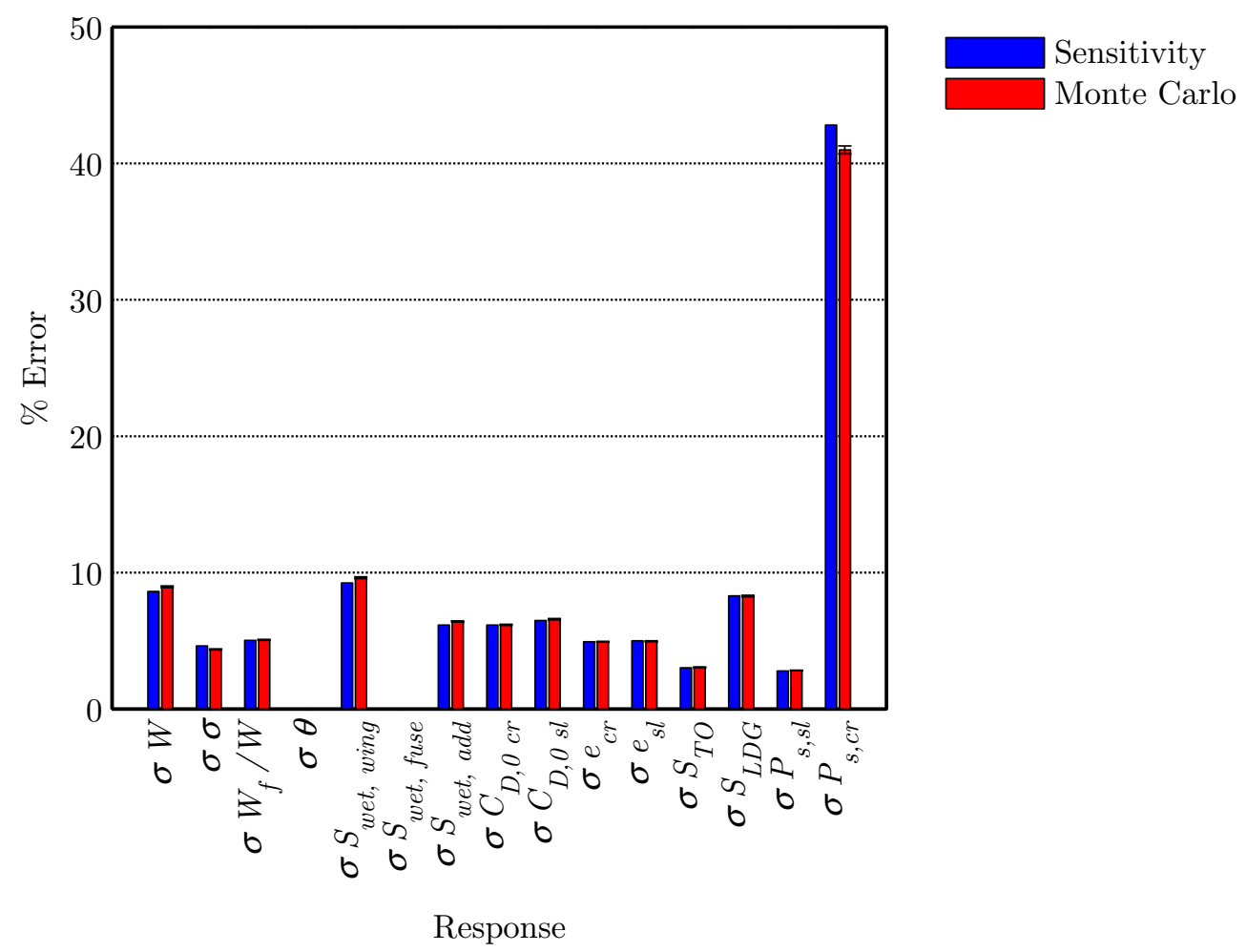

Figure 6. Introductory error verification

For the Monte Carlo study, Gaussian probability distributions with zero mean and the specified variance were assumed for each of the error sources. A random sample of 10,000 cases were analyzed, and the complete system was brought to convergence for every case.

In general, the agreement between the two approaches is very good, and the sensitivity approach is verified as an approximate model of the propagation of error through a complex system. The $\sigma P_{s, c r}$ result shows the greatest disparity, clearly beyond that which can be attributed to the finite sample size; this disparity is due to the linearization introduced by the sensitivity approach.

The Monte Carlo verification analysis took about five minutes to complete for a single point in the design space. Each update of the fidelity trade environment would require approximately $500 \mathrm{such}$ analyses. The sensitivity approach allows what would otherwise be a very expensive calculation to be performed in an interactive manner, thereby making the fidelity trade environment possible. The comparative nature of the results displayed by the fidelity trade environment do not require extreme accuracy, and the approximations introduced by the linearization are believed by the author to be acceptable.

The gray band surrounding the system response in Figure 3 represents the system propagated error throughout the design space.

The impact of error on the design space as represented by the constraint diagram is shown in Figure 4(a). Note that the error on the landing field length constraint has dramatic impact on the choice of the design point of the vehicle. Not only have the constraint locations with the consideration of error changed, but the constraints active in choosing a design point have changed. Before, the design point was constrained by specific excess power at cruise and takeoff field length; afterward, the design point was constrained by specific excess power at cruise and landing field length.

When faced with the constraint diagram depicted in Figure 4(a), the designer may desire to reduce the error impacting his choice of design point. His first step may be to improve the landing field length 
estimation. Referring to Figure 5, we note that the primary source of error for landing field length is the error in maximum lift coefficient. The designer may then choose to perform a wind tunnel test to reduce the error on this quantity. The error levels for the system including reduced $C_{L, \max }$ error for landing are listed below in Table $3(\mathrm{~b})$.

The error breakdown corresponding to the system with reduced $C_{L, \max }$ error is shown in Figure 7. Note that while the error in takeoff and landing distance were significantly reduced, no other quantities were impacted.

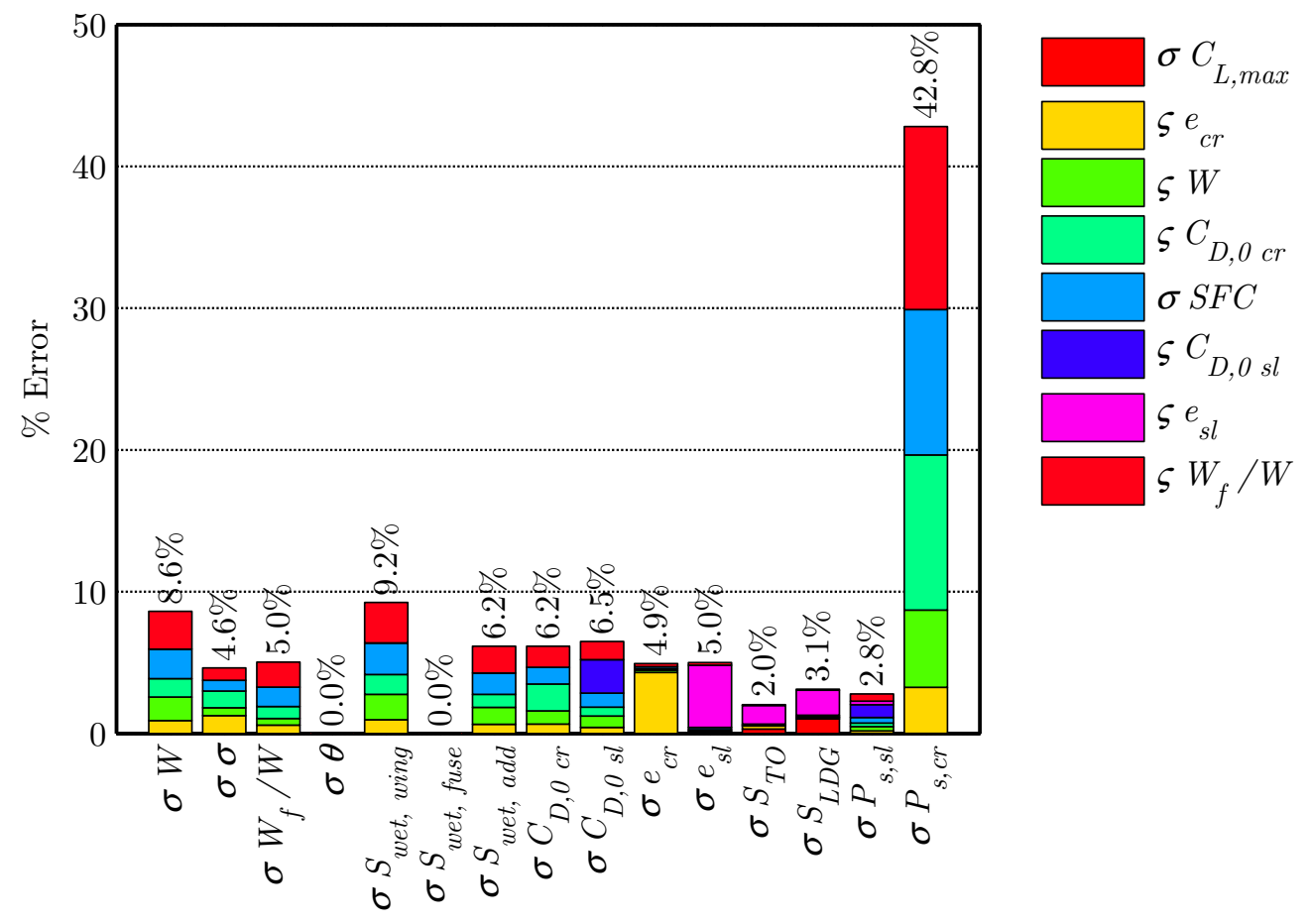

Response

Figure 7. Error breakdown with reduced landing error

Figure 4(b) demonstrates the effectiveness of reducing the error in $C_{L, \max }$ on the design point selection. While the active constraints have still changed, the impact of error on choosing the design wing loading has been minimized.

Examination of Figure 4(b) demonstrates that the impact of error on the design point is now primarily due to error in specific excess power at cruise. Reducing this error is now the designer's priority. The error breakdown shown in Figure 7 indicates which error sources have the greatest impact on cruise specific excess power. The designer may then choose to reduce the error in some of these quantities as listed in Table 3(c). The cruise drag coefficient estimate $C_{D, 0} \mathrm{cr}$ could be improved through a wind tunnel test, while an improved estimate of the cruise specific fuel consumption $S F C$ could be requested from the engine manufacturer. An improved mission model could improve the fuel burn calculation $W_{f} / W$.

The error breakdown corresponding to the system for reduced cruise error is shown in Figure 8. While still showing the largest percent error, error in cruise excess power has been reduced enough to warrant changing the scale of the error breakdown.

Figure 4(c) demonstrates the impact of reducing cruise error on the design point selection. As intended, the impact of error on choosing the design thrust loading has been minimized.

Interpretation of Figure 4(c) may show that the level of error impacting the design point is acceptable to the designer. Once a design is selected, the primary purpose of an aircraft systems study is to estimate the size of the vehicle. The takeoff gross weight of an aircraft is a primary driver of the manufacturing, operating, maintenance, and other costs of the vehicle. In the absence of further analysis, minimization of aircraft weight tends to minimize cost. Examination of the error breakdown shown in Figure 8 shows 


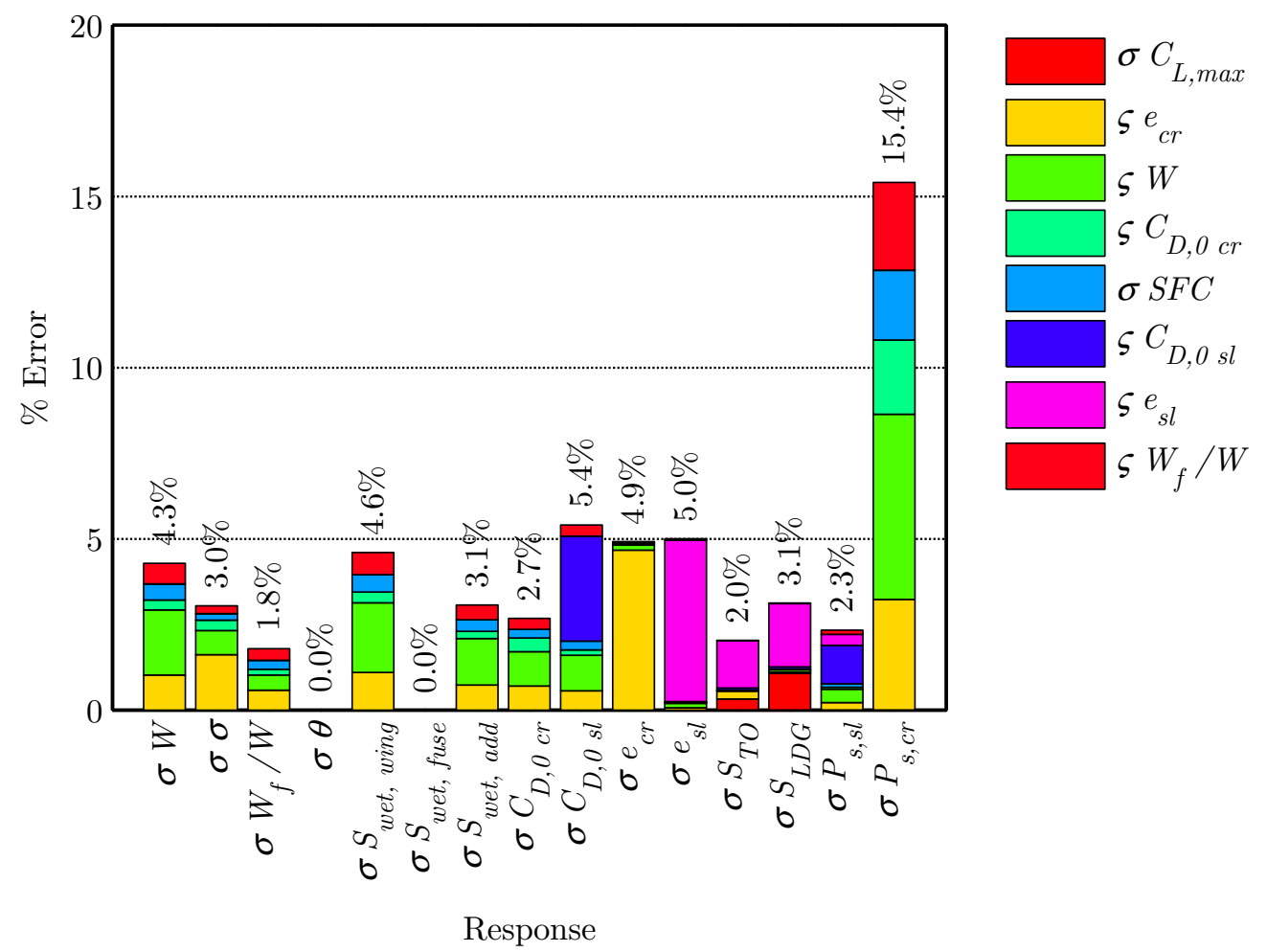

Figure 8. Error breakdown with reduced cruise error

that the biggest remaining contributor to error in takeoff gross weight is the weight calculation itself. The designer may then choose to reduce the error in the weight calculation as listed in Table 3(d). It is worth pointing out the counterintuitive result of a propagated error of $4.3 \%$ in weight when the local error in the weight analysis alone is $5.0 \%$. This is due primarily to the coupling structure of the system, which results in a weight error which is "stable". Error stability is further discussed in the next section.

The error breakdown corresponding to the system with reduced weight calculation error is shown in Figure 9. As intended, the error in the takeoff gross weight estimation has been significantly reduced from $4.3 \%$ to $2.5 \%$. The corresponding system explorer with the error distribution throughout the design space is shown in Figure 10; in this figure, the gray bands surrounding the takeoff and landing distance responses in Figure 3 have virtually vanished. Similarly, the corresponding constraint diagram is shown in Figure 4(d).

In this scenario, the designer was faced with a complex system incorporating a variety of error sources. His first actions were to reduce the sources of error contributing to uncertainty in the choice of the design point. Once a design point could be chosen with confidence, his next action was to reduce the sources of error contributing to the most important system level metrics. In so doing, some significant sources were allowed to remain, being quantitatively determined to be unimportant to the decision making process undertaken by the designer.

\section{Error Stability}

Equation 2 can be used to propagate an arbitrary combination of sources of error through an arbitrarily complex system. The behavior of error in a complex system can sometimes be counterintuitive. In order to understand this behavior, it is instructive to examine some simplified situations in the context of Equation 2.

First, consider the situation where there is no error in any of the input quantities. In this situation, the $\sigma_{\mathbf{x}}^{2}$ vector is a zero vector, and Equation 2 simplifies to Equation 3.

$$
\sigma_{\mathbf{f}}^{2} \approx \mathbf{M}^{-12} \varsigma_{\mathbf{f}}^{2}
$$




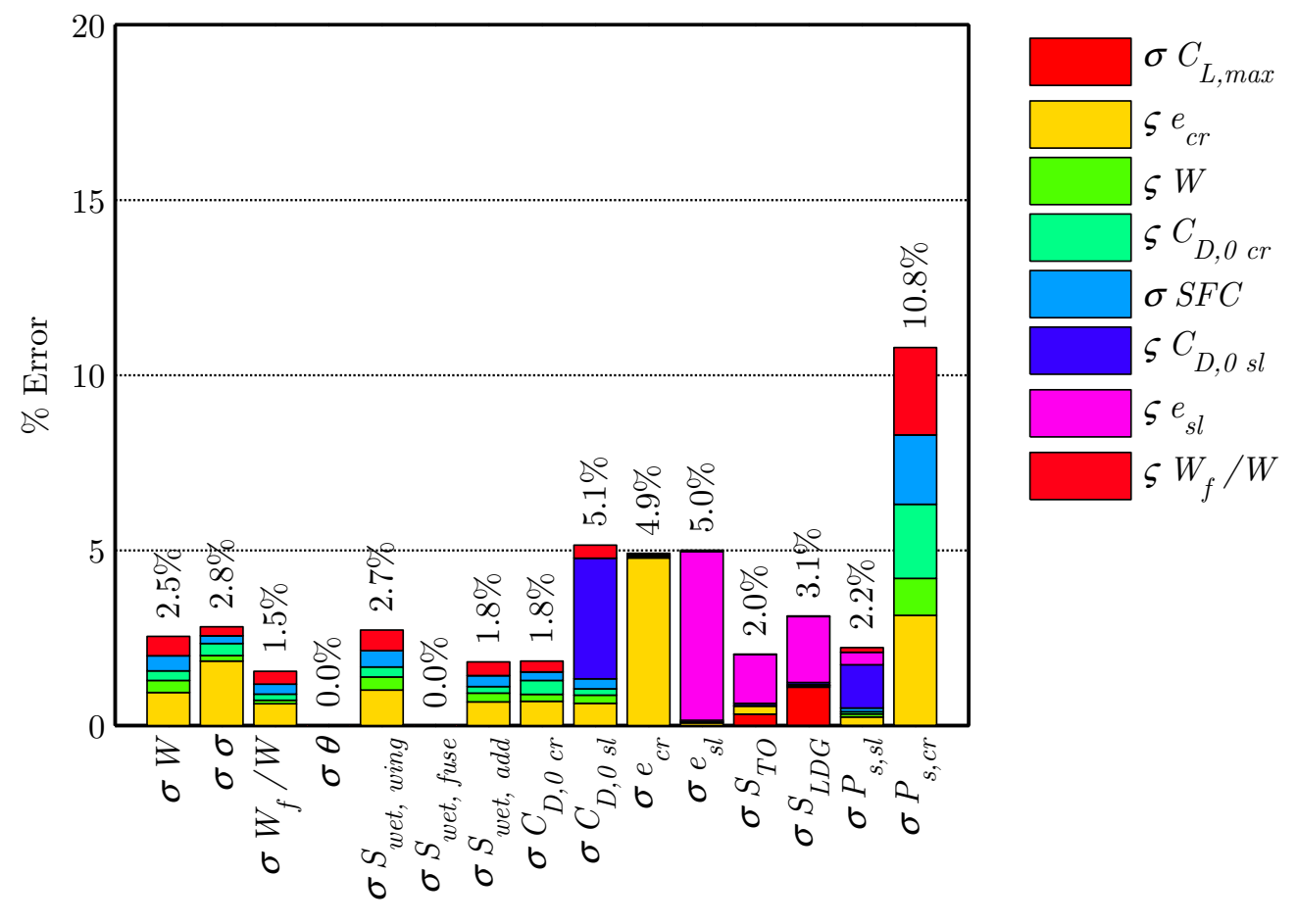

Response

Figure 9. Error breakdown with reduced weight error

Further, consider the situation where only one error source is introduced at any time. In this situation, the squared vector of error sources $\left(\varsigma_{\mathbf{f}}^{2}\right)$ has only one nonzero term.

In this situation, the matrix-vector multiplication simplifies to a vector-scalar multiplication, where the vector corresponds to the appropriate row of the matrix, and the scalar corresponds to the nonzero term of the error source vector. Of course, the vector-scalar multiplication does not have the dot-product-like implied summation of terms present in a matrix-vector multiplication. Furthermore, without the implied summation, we can safely take the square root of both sides of the equation.

Therefore, in the situation of a solitary source of error, the propagation of error loses the Pythagorean sum. This situation matches intuition; after all, when one side of a right triangle is of vanishingly small length, the other side's length is equal to the length of the hypotenuse.

Furthermore, inspection of the diagonal of the $\mathbf{M}^{-1}$ matrix yields insight into the stability of an error source in the complex system. When an individual source of error is introduced to a quantity in a system, the output error level of that quantity is determined by the corresponding element of the diagonal of the $\mathbf{M}^{-1}$ matrix. If the corresponding term of the diagonal is greater than one, the error in the system output will be greater than the error introduced. However, if the corresponding term of the diagonal is less than one, the error in the system output will be less than the error introduced. The term error stability is used in an analogy to aircraft stability; a stable error will tend to be damped by the system while an unstable error will tend to be amplified by the system.

The counterintuitive decay of an error source in a complex system can be understood in terms of system stability. For some quantities, the system tends to amplify an error source; whereas, for other quantities, the system tends to diminish an error source. Whether any particular error source grows or decays is dependent on the overall system behavior at that point in the design space.

Whether a source of error grows or decays is determined by its diagonal of the $\mathbf{M}^{-1}$ matrix. Of course, the diagonal elements of the $\mathbf{M}$ matrix are all equal to one. Consequently, whether the diagonal terms in the $\mathbf{M}^{-1}$ matrix are greater or less than one is determined by the off diagonal terms of the system sensitivity matrix $(\mathbf{M})$.

The counterintuitive phenomenon of decaying error sources was encountered in the transport aircraft 


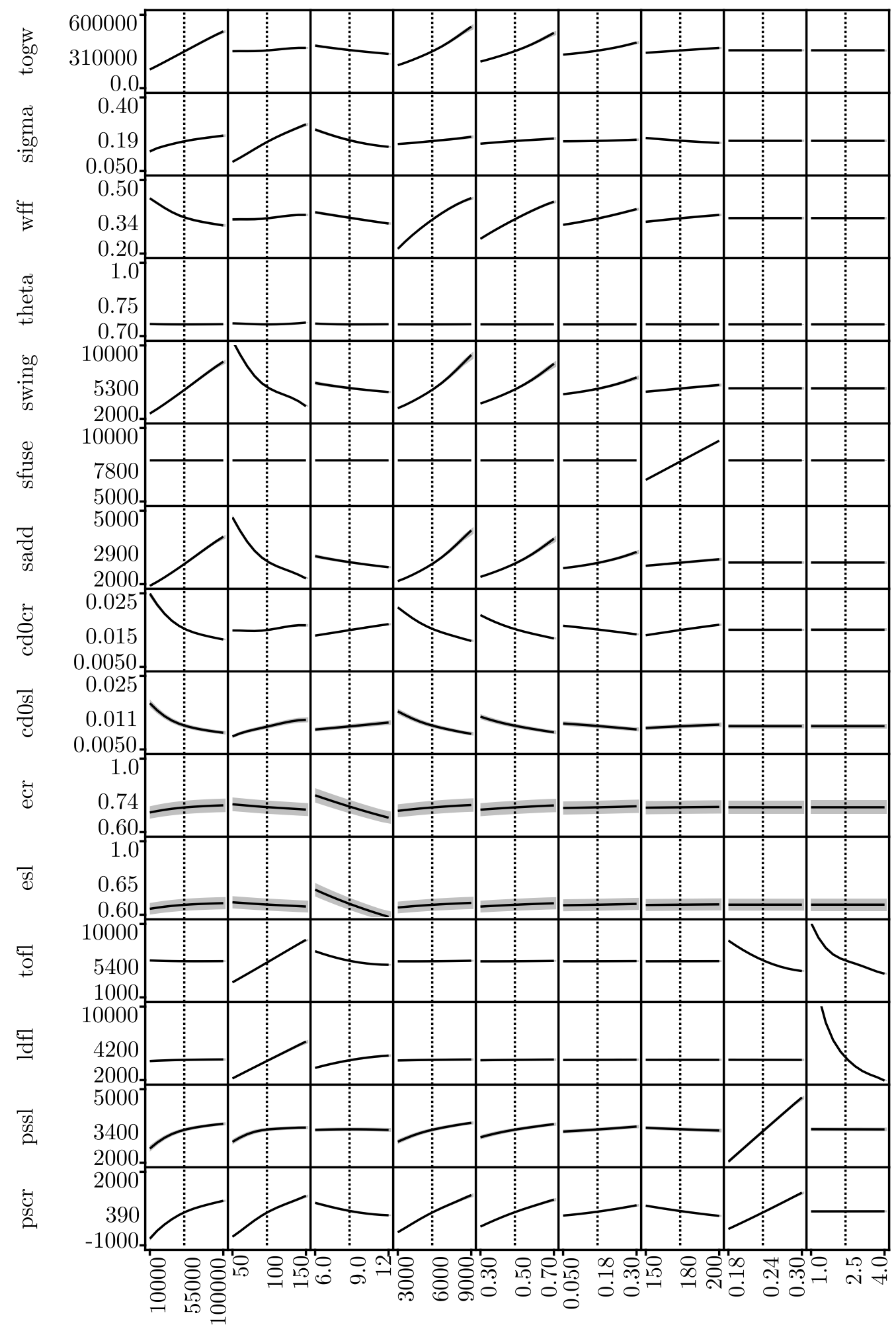

wp wos ar range sfccr wffadd lfuse tow clmax

Figure 10. Aircraft design space with reduced weight error 
example. The diagonal of the system sensitivity inverse $\left(\mathbf{M}^{-1}\right)$ matrix at the design point was listed in Table 4(a). These values are reported to five decimal places to facilitate inspection of their detailed numerical behavior, not to imply an extended level of confidence or significance in their precision.

Table 4(a) reveals interesting insight into the behavior of the complex system. First, note that six of the terms are exactly equal to one. These six output quantities do not participate in coupling. The final four quantities $\left(S_{T O}, S_{L D G}, P_{s, s l}\right.$, and $\left.P_{s, c r}\right)$ are pure output quantities, i.e. no other quantity depends on them. The other two quantities with diagonals equal to one $\left(C_{D, 0} s l\right.$ and $\left.e_{s l}\right)$ are only depended on by the pure output quantities. All quantities with a diagonal not equal to one participate in the coupling of the system.

Two of the terms are nearly equal to one $\left(\theta\right.$ and $\left.S_{\text {wet, fuse }}\right)$. The first, $\theta$, the cruise temperature ratio is only a very weak function of the vehicle size. In fact, $\theta$ should be constant so long as the vehicle cruises in the stratosphere. However, the atmosphere metamodel used has low amplitude wiggles due to the difficulty of fitting a non-smooth function with a function which has infinite continuous derivatives as supplied by this implementation of a Gaussian process. Consequently, $S_{\text {wet, fuse }}$ has a nonzero diagonal term because it is a function of $\theta$.

The seven other terms listed in Table 4(a) are significantly different from one, and participate significantly in the coupling of the system. Of these terms, four are less than one, and three are greater than one. The largest and smallest diagonal terms were selected for further investigation. For each case, a single 1\% error source in the corresponding quantity was introduced and propagated through the system. The system level errors resulting from a $1 \%$ error source in the takeoff gross weight calculation were listed in Table 4(b). Similarly, the system level errors resulting from a $1 \%$ error source in the wing wetted area calculation were listed in Table 4(c).

Table 4. Illustration of error stability

\begin{tabular}{|c|c|c|c|}
\hline \multirow[b]{2}{*}{ Quantity } & \multirow[b]{2}{*}{$\operatorname{diag} \mathbf{M}^{-1}$} & \multicolumn{2}{|c|}{ Error from $1 \%$ error in } \\
\hline & & $\begin{array}{l}\text { (b) } \\
W\end{array}$ & $\begin{array}{c}\text { (c) } \\
S_{w e t, w i n g}\end{array}$ \\
\hline$W$ & 0.70491 & $0.70491 \%$ & $0.25997 \%$ \\
\hline$\sigma$ & 1.07725 & $0.23544 \%$ & $0.24243 \%$ \\
\hline$W_{f} / W$ & 0.70491 & $0.18553 \%$ & $0.16345 \%$ \\
\hline$\theta$ & 0.99862 & $3.20937 \times 10^{-4} \%$ & $3.30466 \times 10^{-4} \%$ \\
\hline$S_{\text {wet,wing }}$ & 1.27893 & $0.75633 \%$ & $1.27893 \%$ \\
\hline$S_{\text {wet }, \text { fuse }}$ & 0.99997 & $5.99710 \times 10^{-5} \%$ & $2.21169 \times 10^{-5} \%$ \\
\hline$S_{w e t, a d d}$ & 1.10643 & $0.50407 \%$ & $0.1859 \%$ \\
\hline$C_{D, 0 \mathrm{cr}}$ & 0.80017 & $0.39653 \%$ & $0.38158 \%$ \\
\hline$C_{D, 0 \mathrm{sl}}$ & 1.0 & $0.3385 \%$ & $0.29492 \%$ \\
\hline$e_{c r}$ & 0.98342 & $3.06309 \times 10^{-2} \%$ & $1.12264 \times 10^{-2} \%$ \\
\hline$e_{s l}$ & 1.0 & $2.70660 \times 10^{-2} \%$ & $9.87298 \times 10^{-3} \%$ \\
\hline$S_{T O}$ & 1.0 & $5.03960 \times 10^{-3} \%$ & $8.95290 \times 10^{-3} \%$ \\
\hline$S_{L D G}$ & 1.0 & $2.20047 \times 10^{-2} \%$ & $2.233383 \times 10^{-3} \%$ \\
\hline$P_{s, s l}$ & 1.0 & $0.14448 \%$ & $0.121507 \%$ \\
\hline$P_{s, c r}$ & 1.0 & $2.24509 \%$ & $2.149289 \%$ \\
\hline
\end{tabular}

As expected, the error in the output quantity corresponding to the error source in each case is equal to $1 \%$ of the corresponding term of the diagonal of the $\mathbf{M}^{-1}$ matrix. In this case, the system tends to shrink errors in the takeoff gross weight calculation, while it tends to amplify errors in the wing wetted area calculation.

\section{Conclusions}

This paper described the use of a fidelity trade environment as applied to a transport aircraft. The fidelity trade environment was used in a successful decision making processes concerning the fidelity of tools 
in complex systems design; this scenario was not possible without the fidelity trade environment. The role of system feedback and coupling in error stability was also investigated.

This research has demonstrated the fidelity trade environment as a decision making tool to help the designer answer questions like: Is the fidelity of a complex systems analysis adequate, or is an improvement needed? If an improvement is needed, how is that improvement best achieved? Where should limited resources be invested for the improvement of fidelity? How does knowledge of the imperfection of a model impact design decisions based on the model? How does this knowledge impact the choice and certainty of the design point? How does it impact the certainty of the performance of a particular design?

\section{References}

${ }^{1}$ McDonald, R. A., Error Propagation and Metamodeling for a Fidelity Tradeoff Capability in Complex Systems Design, Ph.D. thesis, Aerospace Engineering, Georgia Institute of Technology, Atlanta, GA, August 2006.

${ }^{2}$ McDonald, R. A., "Error Allocation in Complex Systems Design," No. AIAA-2006-7130, 11th AIAA/ISSMO Multidisciplinary Analysis and Optimization Conference, Portsmouth, VA, September 6-8 2006.

${ }^{3}$ Baird, D. C., Experimentation: An Introduction to Measurement Theory and Experiment Design, Prentice-Hall Inc., Englewood Cliffs, NJ, 1962.

${ }^{4}$ Taylor, J. R., An Introduction to Error Analysis The Study of Uncertainties in Physical Measurements, University Science Books, Sausality, California, 2nd ed., 1997.

${ }^{5}$ Oberkampf, W. L., Diegert, K. V., Alvin, K. F., and Rutherford, B. M., "Variability, Uncertaintity, and Error in Computational Simulation," HTD-Vol. 375-2, ASME Proceedings of the 7th AIAA/ASME Joint Thermophysics and Heat Transfer Conference, edited by R. S. Amano, B. F. Armaly, T. S. Chen, A. Emery, J. A. Liburdy, D. P. Shatto, N. K. Anand, B. Blackwell, T. Y. Chu, J. Lage, O. Oosthuizen, and K. A. Woodbury, No. H1137B, 1998.

${ }^{6}$ Oberkampf, W. L., DeLand, S. M., Rutherford, B. M., Diegert, K. V., and Alvin, K. F., "Error and Uncertainty in Modeling and Simulation," Reliability Engineering 83 System Safety, Vol. 75, 2002, pp. 333-357.

${ }^{7}$ Sobieszczanski-Sobieski, J., "Sensitivity of Complex, Internally Coupled Systems," AIAA Journal, Vol. 28, No. 1, January 1990, pp. 153-160.

${ }^{8} \mathrm{Du}, \mathrm{X}$. and Chen, W., "Efficient Uncertainty Analysis Methods for Multidisciplinary Robust Design," AIAA Journal, Vol. 40, No. 3, 2002, pp. 545-552. 